\title{
Aurora Kinase-A Regulates Microtubule Organizing Center (MTOC) Localization, Chromosome Dynamics, and Histone-H3 Phosphorylation in Mouse Oocytes
}

\author{
JUN DING, ${ }^{1}$ JASON E. SWAIN, ${ }^{1,2}$ AND GARY D. SMITH ${ }^{1,2,3,4 *}$ \\ ${ }^{1}$ Department of Obstetrics and Gynecology, University of Michigan, Ann Arbor, Michigan \\ ${ }^{2}$ Reproductive Sciences Program, University of Michigan, Ann Arbor, Michigan \\ ${ }^{3}$ Department of Molecular and Integrative Physiology, University of Michigan, Ann Arbor, Michigan \\ ${ }^{4}$ Department of Urology, University of Michigan, Ann Arbor, Michigan
}

\begin{abstract}
SUMMARY
Aurora kinases (AURKs) are conserved serine/threonine kinases, crucial in regulating cell cycle events. Mammalian oocytes express all three Aurk isoforms throughout meiosis, with AurkA being the predominant isoform. Inhibition of all AURK isoforms by pharmacological means disrupts oocyte meiosis. Therefore, AurkA short interfering RNA (siRNA) was performed to silence AurkA gene expression in mouse oocytes and to further assess the function of AurkA during meiosis by analyzing subsequent loss-of -function oocyte phenotypes. Results indicated that AurkA siRNA applied in our experiments specifically knocked down both AurkA gene and protein expression without influencing transcript levels of AurkB/AurkC and other endogenous protein expression, such as GAPDH and ERK-2. AURKA was not essential for resumption of meiosis, but it potentiated oocyte meiotic progression. Knockdown of AurkA led to a significant reduction in the number of oocytes proceeding to metaphase II (MII). AurkA siRNA resulted in abnormal spindle assembly, improper localization of microtubule organizing centers (MTOCs) and misalignment of chromosomes in metaphase I (MI) oocytes. Co-immunoprecipitations demonstrated that AURKA was physically associated with phospho-Histone $\mathrm{H} 3$ ser10 in meiotic oocytes. AurkA siRNA dramatically reduced Histone $\mathrm{H} 3$ ser10 phosphorylation, but not ser28, and resulted in a significant increase of abnormal chromosome segregation in MII oocytes. In conclusion, as a predominant isoform among Aurks in oocytes, AurkA plays critical roles in mouse oocyte meiosis by regulating spindle and chromosome dynamics.
\end{abstract}

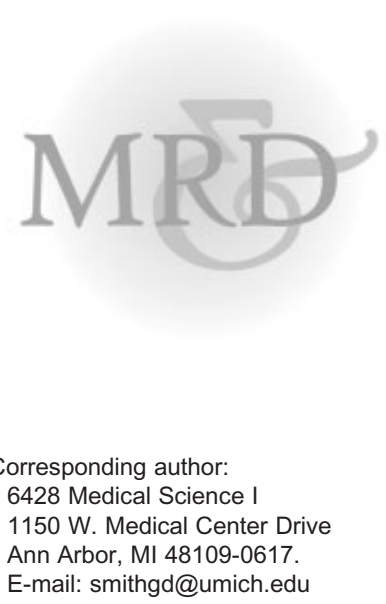

Grant sponsor: NIH, (HD046768)

Mol. Reprod. Dev. 78: 80-90, 2011. (C) 2010 Wiley-Liss, Inc.

\section{INTRODUCTION}

Aurora kinases (AURKs) are an evolutionary conserved serine/threonine kinase family that plays a pivotal role in regulating cell cycle dynamics. In mitosis, AURKs are directly involved in regulation of mitotic entry, centrosome

\footnotetext{
Abbreviations: AURK, aurora kinase; dsRNA, double-stranded RNA; GVI, germinal vesicle-intact; GVBD, germinal vesicle breakdown; IBMX, isobutylmethylxanthine; MI, metaphase I; MII, metaphase II; MTOC, microtubule organizing center; RNAi, RNA interference; RISC, RNA-induced silencing complex; siRNA, short interfering RNA.
} 
maturation and separation, mitotic bipolar spindle assembly, chromosome segregation and cytokinesis (Crane et al., 2003; Ducat and Zhen, 2004; Meraldi et al., 2004). Three AURK isoforms, $A, B$, and $C$, are characterized in mammals based on their distinct subcellular localization and function in the cell cycle (Crane et al., 2003). AURKB localizes to chromosome kinetochores and the midbody during mitosis, and appears essential for chromosome condensation and cytokinesis (Ditchfield et al., 2003; Goto et al., 2003). AURKC is a chromosome passenger protein ( $\mathrm{Li}$ et al., 2004) that associates with AURKB and survivin in mitotically dividing cells (Yan et al., 2005). Finally, AURKA localizes to centrosomes and spindle poles, and is primarily responsible for centrosome maturation and separation, and for bipolar spindle assembly during mitosis (Marumoto et al., 2005; Ulisse et al., 2006). In addition, AURKA regulates other important mitotic events, such as spindle checkpoint, mitotic entry, kinetochore function, cytokinesis, cell asymmetric division and cell fate determination (Barr and Gergely, 2007). Both depletion and overexpression of Aur$k A$ results in abnormal mitosis, and leads to genomic instability, aneuploidy, and tumor formation (Anand et al., 2003; Lu et al., 2008).

Though defined roles for AURKs exist for mitotically dividing cells, their functional roles during oocyte meiosis are just emerging. Accumulating evidence demonstrates that AURKs regulate meiotic progression, spindle dynamics and chromosome remodeling in both lower vertebrate and mammalian oocytes (Swain et al., 2008; Uzbekova et al., 2008; Lane et al., 2010). All three Aurk isoforms are expressed in mammalian oocytes throughout meiotic progression (Swain et al., 2008; Uzbekova et al., 2008; Shuda et al., 2009). Inhibition of all three oocyte AURKs using inhibitor ZM447439 results in meiotic arrest at a metaphase I (MI)like stage, displaying irregular spindle formation, inhibition of histone $\mathrm{H} 3$ phosphorylation and abnormal chromosome condensation (Swain et al., 2008; Eichenlaub-Ritter, 2009). However, little information exists regarding the function of individual Aurks during mammalian oocyte meiosis. A recent paper using mRNA microinjection describes a role for AURKC in mouse oocytes (Yang et al., 2010) and two papers have attempted to delineate roles for AURKA. Yao et al. (2004) utilized antibody neutralization in mouse oocytes and found that AURKA inhibition interfered with oocyte meiotic resumption and distorted $\mathrm{Ml}$ spindle organization. Saskova et al. (2008) reported that overexpression of AurkA led to the formation of an abnormal MI spindle, while reduction of AurkA via long double-stranded RNA (dsRNA) interfered with oocyte meiotic resumption and spindle assembly. Although AURKA was demonstrated to co-localize with MTOCs and chromosomes during oocyte meiosis (Yao et al., 2004; Saskova et al., 2008), no detailed information regarding the impact of AurkA on MTOCs and chromosome dynamics during oocyte meiotic progression were reported, and the specificity of previously used knockdown approaches is also questionable.

One method to determine function of specific intracellular regulators is RNAi. RNAi is a procedure that leads to gene silencing through degradation of the target transcript
(Fire et al., 1998; Hammond et al., 2000). This mechanism is initiated by dsRNA homologous to the gene being silenced. The dsRNAs are processed by an enzyme named Dicer to generate duplexes of $21 \mathrm{nt}$ with $3^{\prime}$-overhangs called small interfering RNA, or siRNA. The siRNA assembles into RNA-induced silencing complexes (RISCs) and directly mediates the sequence-specific mRNA degradation (Meister and Tuschl, 2004; Mello and Conte, 2004). Though fully grown oocytes progressing through meiosis historically have been thought to be transcriptionally inert, it has been confirmed that synthetic siRNA can be delivered manually into oocytes during this developmental period to silence specific genes (Svoboda et al., 2000; Wianny and Zernicka-Goetz, 2000; Wang et al., 2007; Solc et al., 2008). However, validation of specificity is crucial before specific functions can be attributed to various genes, especially in closely related genes such as the three Aurk isoforms.

To begin to delineate Aurk isoform-specific function during mammalian oocyte meiosis, AurkA siRNA was microinjected into mouse oocytes to silence gene expression, and specificity of the approach was thoroughly validated to ensure no impact on other Aurk isoforms. The functions of AurkA during mouse oocyte maturation was then determined by examining loss-of-function phenotypes, focusing on microtubule organizing center (MTOC) arrangement, spindle organization, and chromosome dynamics.

\section{RESULTS}

\section{AurkA siRNA Specificity and the Influence on Oocyte Meiotic Progression}

AurkA siRNA specificity was determined on both AurkA transcriptional and translational levels in germinal vesicleintact (GVI) oocytes just before re-initiation of meiosis with IBMX withdrawal after $24 \mathrm{hr}$ incubation. The real-time PCR analysis demonstrated that, in comparison to control oocytes, AurkA siRNA could specifically knockdown AurkA transcript levels 59- to 67-fold (upper 98\% efficiency) without significantly affecting the other two Aurk isoforms (AurkB and AurkC) (Fig. 1A); and Western blot analysis further confirmed that AURKA protein expression levels were dramatically reduced in oocytes before meiotic resumption by AurkA siRNA, without significantly influencing other oocyte endogenous protein expression, represented by GAPDH and ERK2 assessments (Fig. 1B).

To determine if AurkA knockdown impacts oocyte meiotic resumption and progression, oocytes undergoing GVBD and development to metaphase II (MII) in control and AurkA siRNA groups were compared at 2 and $17 \mathrm{hr}$ after release from meiotic arrest, respectively. Results demonstrate that AurkA knockdown had no significant influence on oocyte meiotic resumption or GVBD. Rates of GVBD were $94 \%, 95 \%$, and $90 \%$ for buffer $(n=358)$, non-targeted siRNA $(\mathrm{n}=132)$, and AurkA siRNA $(\mathrm{n}=355)$, respectively (Table 1). However, AurkA knockdown significantly reduced the rate of MII development $(58 \% ; P<0.05)$ 


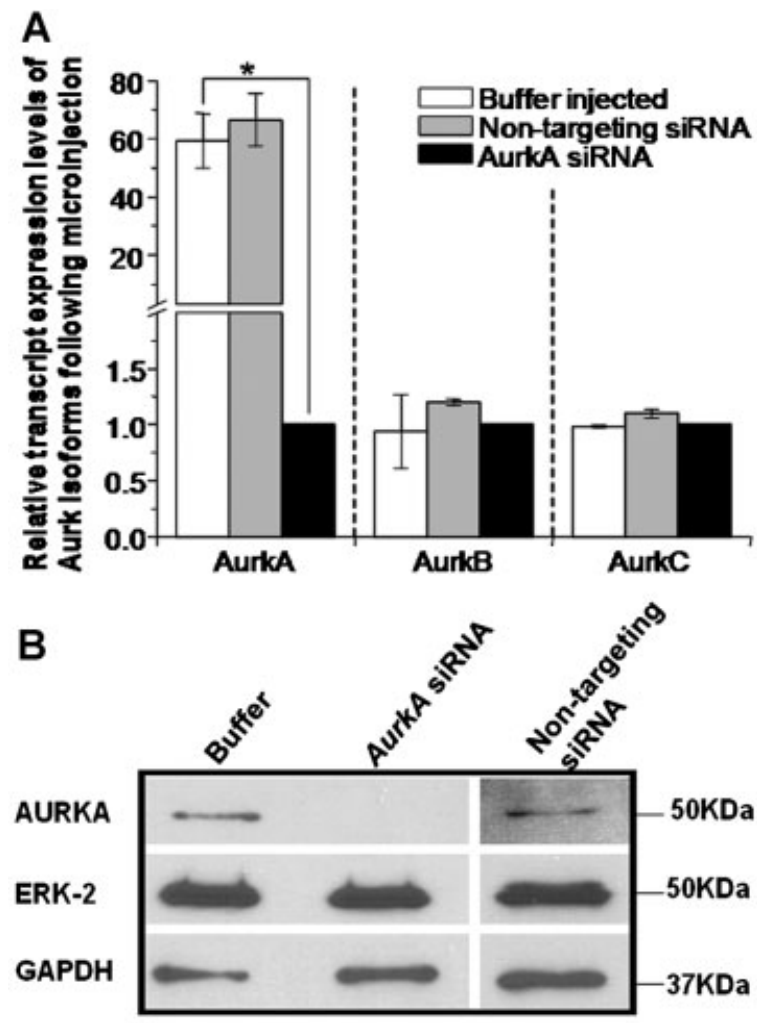

Figure 1. A: AurkA transcript level was significantly reduced in AurkA siRNA injected oocytes compared with buffer and non-targeting siRNA injected controls $(P<0.01)$, whereas AurkB and AurkC transcript levels are not significantly influenced. Data are presented as mean \pm SEM and statistical significance was determined using unpaired Student's $t$-test, $P<0.01$. Oocyte samples were collected at the GVI stage just before re-initiation of meiosis with IBMX withdrawal. B: Representative Western blot analysis demonstrating that AURKA protein expression was significantly knocked down by AurkA siRNA, but not with non-targeting siRNA or buffer injection. Equal numbers of GVI oocytes were loaded ( $n=130$ each lane). Experiments were repeated three times.

compared to buffer $(80 \%)$ and non-targeting siRNA (81\%) microinjection (Table 1).

\section{MTOC Localization, Spindle Assembly, and Chromosome Alignment in Metaphase I Oocytes}

Confocal microscopic analysis showed that significantly more $(86 \%)$ of control MI oocytes $(n=30)$ possessed regular spindles with aligned chromosomes and bipolar
MTOCs (Fig. 2A) than AurkA siRNA MI oocytes (32\%, $\mathrm{n}=35$ ). Approximately $68 \%$ of AurkA siRNA oocytes showed irregular MI spindles with their MTOC improperly localized and their chromosomes misaligned (Fig. 2B, $P<0.01$ ). Unlike control oocytes containing irregular $\mathrm{MI}$ spindles with the random appearance of mislocalized MTOCs or scattered chromosomes or both improperly localized MTOCs and chromosomes, AurkA siRNA oocyte containing irregular spindles normally accompanied misaligned chromosomes and improperly localized MTOCs. Our additional analysis examining structural components of MTOCs through co-localization of two major components, $\gamma$ -tublin and pericentrin, further confirmed that AurkA knockdown significantly disrupted the bipolar localization of MTOCs and resulted in MTOCs scattered around the spindle area in Ml oocytes (Fig. 2C).

\section{Oocyte Chromosome Segregation}

AurkA knockdown led to a significant increase in MII oocytes with abnormal chromosome segregation $(42 \%$ total; $34 \%$ hyperploidy $+8 \%$ hypoploidy, $\mathrm{n}=38$ ) compared to controls ( $25 \%$ total; $18 \%$ hyperploidy $+7 \%$ hypoploidy, $\mathrm{n}=56, P<0.05$; Fig. $3 \mathrm{~A}$ ), and resulted in oocyte aneuploidy (Fig. 3B).

\section{AURKA and Phospho-Histone H3 ser10 During Meiosis and Mitosis}

To identify a substrate for AURKA that may explain the observed phenotypic defects following siRNA and to determine if AurkA can specifically influence chromosome dynamics up to MII, we analyzed oocyte histone $\mathrm{H} 3$ phosphorylation following AurkA siRNA. Results demonstrated that AurkA knockdown inhibited histone $\mathrm{H} 3$ phosphorylation at ser-10, but had no effect on the phosphorylation on the ser-28 (Fig. 4A,B).

To further confirm that AURKA is directly involved in the histone $\mathrm{H} 3$ phosphorylation under physiological conditions, coimmunoprecipitation using phospho-histone $\mathrm{H} 3$ ser 10 antibody was performed in both meiotic oocytes and mitotic KGN cells. Our results demonstrate that AURKA can be specifically coimmunoprecipitated by phospho-histone $\mathrm{H} 3$ ser10 antibody in both mitotic KGN cells (Fig. 4C) and in vivomatured MII oocytes (Fig. 4D), while other proteins, like $\beta$ tubulin, could not be coimmunoprecipitated under the same experimental condition. Since knockdown of AURKA caused inhibition of histone $\mathrm{H} 3$ ser10 phosphorylation, we concluded that AURKA acts as a histone $\mathrm{H} 3$ ser 10 kinase in oocytes.

TABLE 1. Comparison of Germinal Vesicle Breakdown and MII Development in Control and AurkA Knockdown Oocytes

\begin{tabular}{lcc}
\hline & $\begin{array}{c}\text { GVBD } \pm \text { SEM (\%) } \\
\text { 2hr after meiotic resumption }\end{array}$ & $\begin{array}{c}\text { MII } \pm \text { SEM (\%) } \\
\text { 17 hr after meiotic resumption }\end{array}$ \\
\hline Buffer control oocytes $(\mathrm{n}=358)$ & $94 \pm 1.7^{\mathrm{a}}$ & $80 \pm 3.0^{\mathrm{b}}$ \\
Nontargeting siRNA oocytes $(\mathrm{n}=132)$ & $95 \pm 0.84^{\mathrm{a}}$ & $81 \pm 1.6^{\mathrm{b}}$ \\
AurkA siRNA oocytes $(\mathrm{n}=355)$ & $90 \pm 1.7^{\mathrm{a}}$ & $58 \pm 3.0^{\mathrm{c}}$ \\
\hline
\end{tabular}

All data were averaged and based on at least three independent experiments and expressed as a mean \pm SEM. Data were statistically analyzed by unpaired Student $t$-test. Different superscripts $(a, b$, and $c)$ within a column indicate significant differences between treatments, $P<0.05$. 


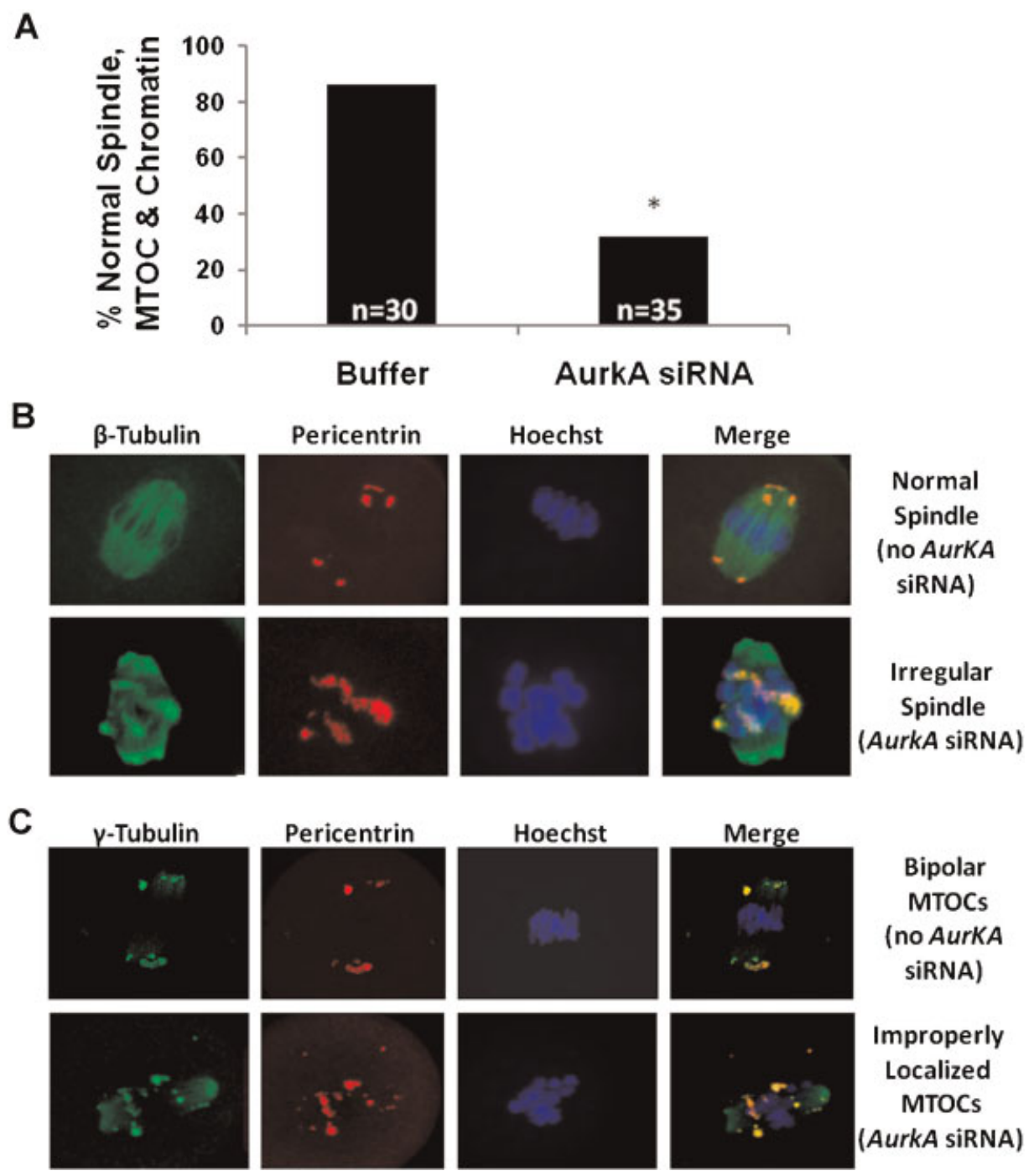

Figure 2. A: AurkA knockdown in mouse oocytes results in a significant reduction in normal spindle and chromatin formation/alignment in MI oocytes (86\% vs. 32\%). Statistical significance was determined using Chi-square analysis, $P<0.01$. B: Representative micrographs resulting from confocal microscopic analysis demonstrating AurkA knockdown lead to improper localization of MTOCs (pericentrin, red), misaligned chromosomes (Hoechst, blue) and irregular shaped spindles ( $\beta$-tubulin, green) in $\mathrm{Ml}$ oocytes cultured for $7 \mathrm{hr}$. C: Additional analysis on impact of AurkA siRNA on MTOC structure. Representative micrographs using confocal microscopic analysis examining co-localization of key structural proteins, $\boldsymbol{\gamma}$-tubulin (green) and pericentrin (red), with and without AurkA siRNA.

\section{DISCUSSION}

AURKA has been localized to nuclei in GV-intact mouse oocytes; co-localized with MTOCs and condensing chromosomes at prophase oocytes; found at the spindle pole, MTOCs and condensed chromosomes in MI oocytes (Yao et al., 2004; Saskova et al., 2008); and further concentrated to spindle poles at MII oocytes (Yao et al., 2004). Recent comparative studies on levels of three Aurk isoform transcripts demonstrated that AurkA was the predominant form in meiotic competent bovine and mouse oocytes, and its transcript levels were much higher than AurkB and AurkC
(Swain et al., 2008; Uzbekova et al., 2008; Shuda et al., 2009). These data suggest a crucial role of AurkA among the three isoforms in regulating the progression of oocyte meiosis in mammals.

In this study, mouse AurkA sequence-specific siRNA was utilized to assess the function of AurkA during mouse oocyte meiosis. Importantly, results demonstrated that AurkA siRNA could specifically inhibit AurkA gene transcription and translation without influencing AurkB, AurkC transcript levels. Furthermore, because no AURKB or AURKC antibodies could be found to give reliable results in mouse oocytes, we also utilized GAPDH and ERK-2 antibodies as 


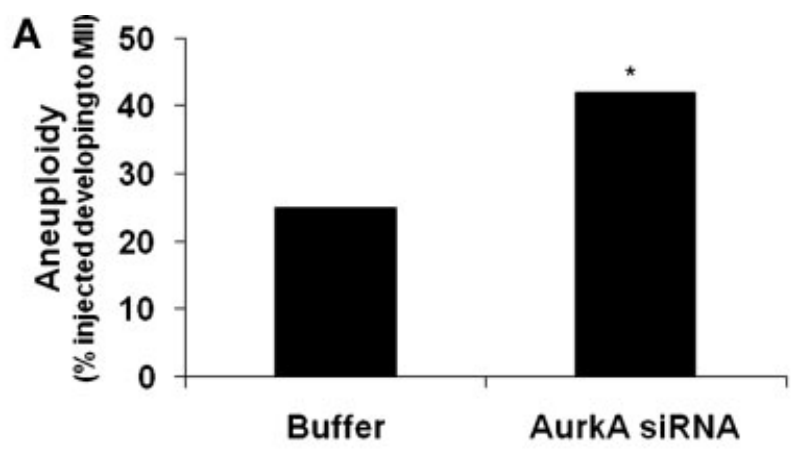

B
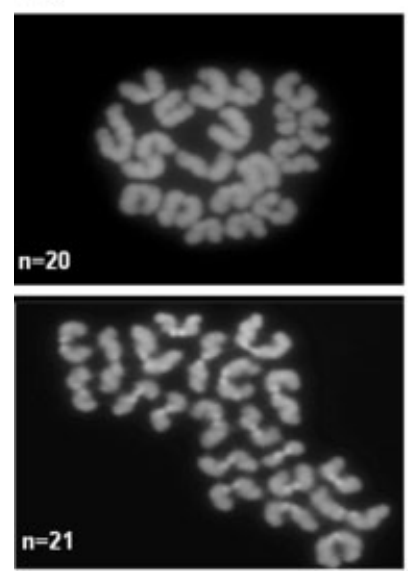
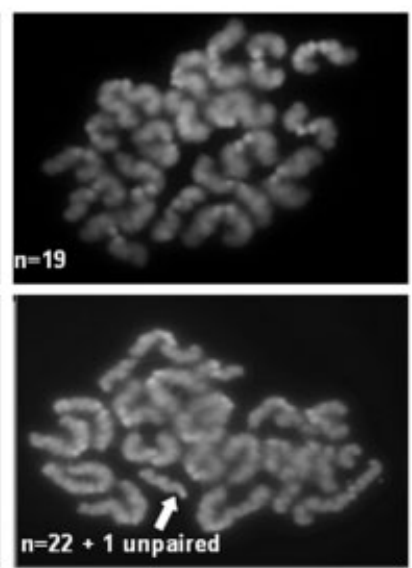
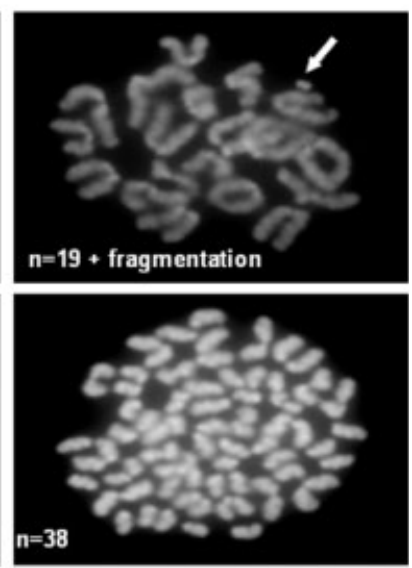

Figure 3. A: AurkA knockdown significantly inhibits normal chromosome segregation in oocytes, as $42 \%$ of MII oocytes displayed abnormal chromosome complement compared to $25 \%$ of controls. Statistical significance was determined using Chi-square analysis, $P<0.05$. B: Representative chromosome spread micrographs demonstrating the aneuploidies observed in Mll oocytes cultured for $17 \mathrm{hr}$ after AurkA knockdown ( $\mathrm{n}=$ number of chromosomes in image).

indicators to verify that endogenous protein levels in our experiment were not impacted by AurkA siRNA. In contrast, two prior studies examining AurkA function in oocytes using antibody neutralization (Yao et al., 2004) and long double strand AurkA RNAi (Saskova et al., 2008), failed to ensure that the antibody and dsRNAi applied in their experiments were Aurk isoform-specific and had no impact on overall endogenous protein expression. For instance, antibody neutralization studies utilized rabbit IgG injected oocytes as controls, but no further experiments were performed to confirm the specificity of AurkA antibody (Yao et al., 2004) while AurkA RNAi studies used GFP dsRNA as controls but did not confirm whether AurkA dsRNA impacted transcript levels of Aurk B and C (Saskova et al., 2008). Lack of specificity in prior studies may be one explanation for observed differences between studies. Use of rescue studies utilizing AurkA, similar to those conducted with AurkB (Shuda et al., 2009), could help validate findings.

Developmental data demonstrated that AurkA is not essential for spontaneous oocyte meiotic resumption (e.g., GVBD). Though both AurkA transcript and protein in GVI oocytes before meiotic resumption were significantly reduced after $24 \mathrm{hr}$ AurkA siRNA process, oocyte meiotic resumption/GVBD did not differ significantly from controls.
This result is in agreement with the findings that inhibition of bovine and mouse oocyte AURKs with inhibitors VX680 or ZM447439, which inhibit all AURKs, had no significant impact on oocyte GVBD (Swain et al., 2008; Uzbekova et al., 2008). This is also well supported by the recent report that overexpression of AurkA could not override the inhibitory effect on oocyte meiotic resumption caused by high cAMP level (Saskova et al., 2008), while other essential factors (cdc25A and cdc25B) could promote GVBD under similar culture conditions (Solc et al., 2008). However, these results are in contrast to the previous work presented by Yao et al. (2004) and Saskova et al. (2008) who demonstrated that microinjecting AURKA antibody or AurkA long double strand RNA in mouse oocytes inhibited oocyte GVBD. As mentioned, variations in technique or lack of specificity of antibody or RNAi could explain these differences.

Though AurkA knockdown had no impact on meiotic resumption/GVBD at $2 \mathrm{hr}$, AurkA siRNA did lead to a significant reduction in the number of oocytes proceeding to MII and extruding the first polar body after $17 \mathrm{hr}$ of in vitro maturation. This result is consistent with other studies examining roles for all Aurks or AurkA in oocytes (Yao et al., 2004; Saskova et al., 2008; Swain et al., 2008; Uzbe- 


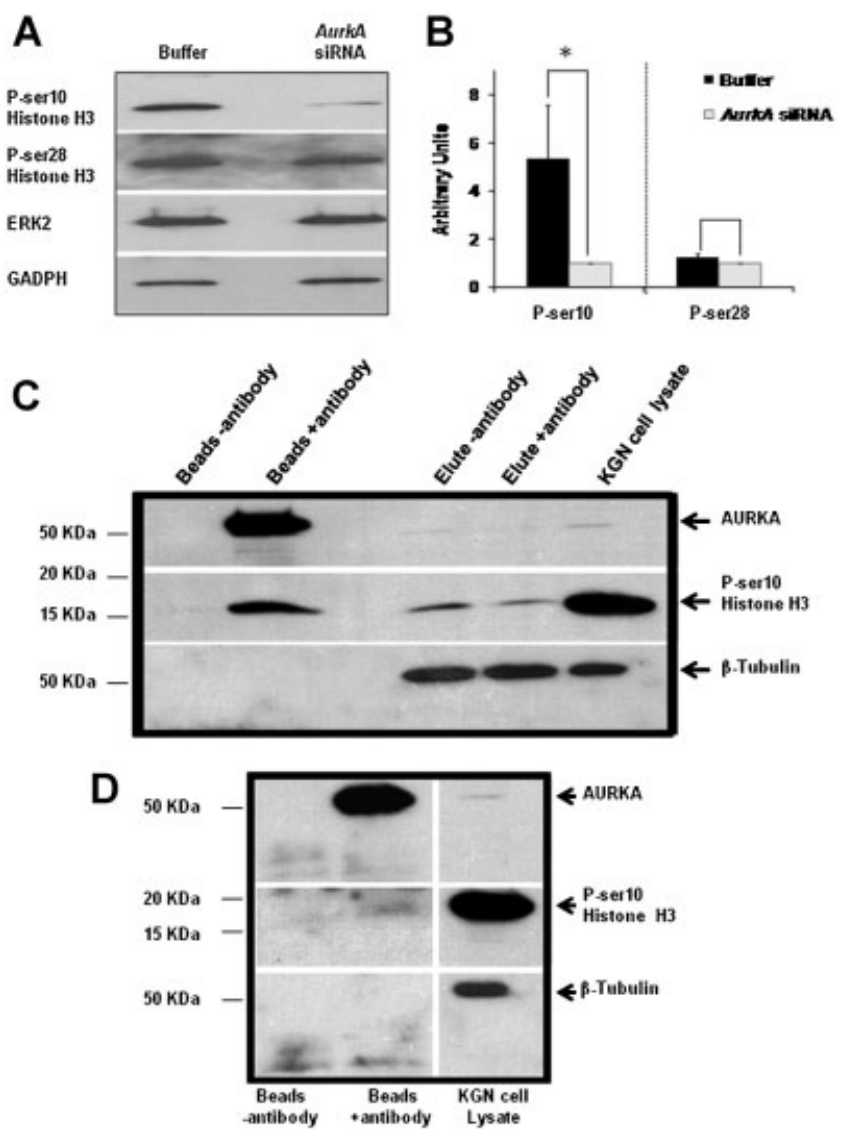

Figure 4. A: Representative Western blot and (B) desitometric analysis demonstrating that AurkA knockdown significantly inhibited the phosphorylation of histone $\mathrm{H} 3$ at ser-10, but not at ser-28. Equal numbers of oocytes $(n=120)$ were loaded in each lane and the membrane was stripped and re-probed for ERK-2 and GAPDH. Experiments were repeated three times. C,D: Representative co-immunoprecipitation results demonstrating that AurkA can be specifically co-immunoprecipitated by phospho-histone H3 ser10 antibody in (C) KGN cells and (D) MII oocytes ( $n=283 /$ lane), while $\beta$-tubulin cannot.

kova et al., 2008). This inhibition may be due to direct effects on chromosomes or to regulation of the meiotic spindle and its components during earlier stages of meiosis, such as microtubules or MTOCs.

Previous reports indicate that AurkA directly regulates centrosome functions in mitotic cells (Crane et al., 2003; Barr and Gergely, 2007). In mouse oocytes, there are no centrosome structures with centrioles, rather MTOCs are reported to function like centrosomes (Schuh and Ellenberg, 2007). AURKA co-localizes with MTOCs during the entire meiotic process in mouse oocytes (Yao et al., 2004; Saskova et al., 2008). This tight structural connection suggests that AURKA may also be functionally associated with MTOCs during oocyte meiosis. Our siRNA studies demonstrated that AurkA knockdown disrupts MTOC location in Ml oocytes. To further explore the influence of AurkA on MTOCs, we stained MI oocytes for two MTOC proteins, gamma tubulin and pericentrin, and examined MTOC organization. Our confocal microscopic analysis is the first evidence demonstrating that highly specific AurkA siRNA in mouse oocytes indeed disrupts the proper localization of these key MTOC proteins. Instead of bipolar localization, MTOCs in AurkA knockdown oocytes were scattered in the middle part of the spindle area. Recent studies indicated that MTOC self-organization and polar migration or pole ejection was critical to bipolar spindle assembly in mouse oocytes (Schuh and Ellenberg, 2007). Therefore, this improper localization of MTOCs might cause the aberrant spindle assembly in MI AurkA siRNA oocytes. Interestingly, evidence suggests that AURKA is an activator of Kinesin-5 (Eg-5) (Crane et al., 2003; Barr and Gergely, 2007), and activation of Eg-5 is a critical regulator of pole ejection and spindle bipolarization in mouse oocytes (Schuh and Ellenberg, 2007).

In addition to its localization with MTOCs, AURKA was also found to co-localize with chromosomes during mouse oocyte meiosis (Yao et al., 2004; Saskova et al., 2008). AURKA initially localized in nuclei before meiotic resumption, then associated with condensing chromatin after GVBD (Yao et al., 2004). Like MTOCs, this structural correlation also suggests a potential role of AurkA in regulating chromatin remodeling during oocyte meiosis. In support of this developmental pathway, immunocytochemistry and chromosome spreads demonstrated that AurkA siRNA significantly increased the number of oocytes with abnormal spindles and chromosome segregation or misalignment on the metaphase plate. Though we only examined MI development at $7 \mathrm{hr}$, there is little reason to think that observed alignment defects were simply due to delayed meiosis. No delay was observed in GVBD and observed defects were severe, unlike organizational structuring of the oocyte meiotic spindle at other time points reported in the existing literature.

It should be noted that our chromosome analysis experiment did yield an $\sim 25 \%$ aneuploidy rate in vehicle microinjected control oocytes, which is much higher than those in the naturally in vivo matured MII oocytes (Hunt et al., 2003; Liu et al., 2008). As evidenced by the elevated hyperploidy rate, this high aneuploidy rate is not due to chromosome loss (hypoploidy) that sometimes occurs during the fixation process. Possible explanations for the elevated rates of aneuploidy observed in control oocytes include the use of equine chorionic gonadotropin (eCG) and/or follicle stimulating hormone (FSH) treatment, which has been reported to increase MII oocyte aneuploidy (Sato and Marrs, 1986; Roberts et al., 2005). Furthermore, there may be an impact from the act of microinjection as well as $24 \mathrm{hr}$ IBMX incubation. Recent publications, however, also demonstrated no dramatic impact on oocyte aneuploidy after FSH treatment (Duncan et al., 2009; Chiang et al., 2010; Lane et al., 2010) or even microinjection (Reis et al., 2007). Importantly, our current experiment was carefully controlled, as both control and AurkA siRNA oocytes were manipulated under the same conditions and the rates of aneuploidy in AurkA siRNA MII oocytes were statistically significantly higher 
than those in control oocytes. Thus, we conclude that AurkA siRNA induced MII oocyte aneuploidy.

In an effort to explain how AurkA regulates oocyte chromosome dynamics, histone $\mathrm{H} 3$ phosphorylation was investigated. Inhibition of histone $\mathrm{H} 3$ phosphorylation, especially histone $\mathrm{H} 3$ ser10 and ser28 phosphorylatyion, is essential in controlling chromosome condensation/ segregation in mitotic cells or mammalian oocytes (Wei et al., 1999; Hsu et al., 2000; Bui et al., 2004; Wang et al., 2006; Swain et al., 2007, 2008). Importantly, AURKA is a potent histone $\mathrm{H} 3$ ser10 kinase in mitotic cells (Crosio et al., 2002). Our Western blot analysis revealed that AurkA knockdown significantly inhibited histone $\mathrm{H} 3$ ser 10 , but not ser28, phosphorylation in oocytes. Our coimmunoprecipitation further demonstrate, for the first time, that AURKA specifically interacts with phospho-histone $\mathrm{H} 3$ ser10 in mammalian oocytes. This is interesting, considering that the previous studies using pharmacologic inhibitors of all AURKs inhibited histone $\mathrm{H} 3$ phosphorylation on both ser 10 and ser 28 (Swain et al., 2008). We therefore conclude that AURKA is acting as a histone $\mathrm{H} 3$ ser10 kinase in meiotic oocytes, while either AURKB or AURKC regulate Ser28 phosphorylation. However, it should be noted that both AURKB and AURKC may also play a role in ser10 phosphorylation. AURKB has been shown to regulate both ser10 and ser28 in mitotic cell extracts (Goto et al., 2003), and its localization to condensed chromatin in oocytes suggests a possible role in meiosis as well. While AURKB has been reported in bovine oocytes (Jelinkova and Kubelka, 2006), conflicting results exists in regard to its presence in mouse oocytes (Shuda et al., 2009; Yang et al., 2010). Previous studies attempting to examine AURKB function in oocytes rely on the use of low doses of broad pharmacologic inhibitors, which inhibit all AURKs and thus make it impossible to designate any specific roles for AURKB (Jelinkova and Kubelka, 2006; Shuda et al., 2009; Lane et al., 2010), though over expression of AurkB did rescue defects in chromosome alignment following broad AURK inhibition using ZM447439 (Shuda et al., 2009). Additionally, AURKC was found to regulate histone $\mathrm{H} 3$ ser10 phosphorylation during mouse oocyte meiosis, though the specificity of the AURKC knockdown approach was not validated and the impact on AURKA levels were not examined (Yang et al., 2010). Thus, it is possible that a redundancy system exists, which permit multiple AURKs to regulate ser10 phosphorylation. It is also important to note that other relevant substrates likely exist that help explain observed chromosomal defects following AurkA knockdown. AURKA phosphorylates several other proteins, such as the kinetochore-specific protein CENP-A in mitotic cells due to a sequence motif similarity to the phosphorylation motif of histone $\mathrm{H} 3$ ser10 (Zeitlin et al., 2000). Inhibition of CENP-A phosphorylation resulted in chromosomal misalignment on metaphase plate in mammalian cells (Cleveland et al., 2003; Kunitoku et al., 2003). Future studies examining additional substrates of AURKA may be informative.

In summary, these studies describe functional roles for Aurk $A$ in mouse oocyte meiosis utilizing a highly specific siRNA approach. AurkA did not appear to be essential for oocyte meiotic resumption; however, it did play a crucial role in regulating mouse oocyte meiotic progression, with reduced AURKA levels disrupting spindle assembly, chromosomal alignment, and normal chromosome segregation when protein levels were reduced. This is the first report demonstrating a critical role for AurkA in proper oocyte MTOC organization/localization, as well as being a histone $\mathrm{H} 3$ kinase that regulates histone $\mathrm{H} 3$ activity through ser10 phosphorylation. Because of the apparently high overlap between all 3 Aurk isoforms in their targets and observed phenotypes, future studies must be careful to confirm specificity of knockdown approaches. Future studies will focus on additional substrates of Aurk $A$ involved in regulation of MTOC localization/organization.

\section{MATERIALS AND METHODS}

All procedures described within were reviewed and approved by The University Committee on Use and Care of Animals at the University of Michigan, and were performed in accordance with the Guiding Principles for the Care and Use of Laboratory Animals.

\section{Mouse Stimulation and Oocyte Collection}

Female CF-1 mice (Harlan, Indianapolis, IN), 2021 days old, were primed with 5 IU equine chorionic gonadotropin (eCG) (Sigma, St. Louis, MO), and fully-grown, meiotically competent germinal vesicle intact (GVI) oocytes were collected after $42-44 \mathrm{hr}$ hormone stimulation. Cumulus-enclosed oocytes were isolated by manually rupturing of antral follicles in human tubual fluid + Hepes media (HTFH; Irvine Scientific, Santa Ana, CA) supplemented with $0.3 \%$ bovine serum albumin (BSA) and $40 \mu \mathrm{M}$ isobutylmethylxanthine (IBMX) (Sigma) to maintain meiotic arrest for subsequent siRNA. Oocytes were then denuded, washed, and kept in $\mathrm{HTF} / 0.3 \% \mathrm{BSA} / 40 \mu \mathrm{M}$ IBMX at $37^{\circ} \mathrm{C}, 5 \% \mathrm{CO}_{2}$ and air incubator prior to microinjection.

\section{AurkA siRNA Designing, Synthesis, Preparation, and Microinjection}

AurkA siRNA was designed based on the NCBI mouse AurkA sequence (NM_011497.2) using Dharmacon Customer Chicago, IL siRNA Design Tool and synthesized by Dharmacon (Chicago, IL) siRNA Technologies. 5'ATACGTACCAGGAGACTT Att 3'; 3'ttTATGCATGGTCC ttTATGCATGGTCC T CTGAAT $5^{\prime}$. The nontargeting, scrambled siRNA was purchased from Dharmacon (D001210-01-05). Both AurkA siRNA and nontargeting siRNA were reconstituted in Dharmacon siRNA buffer to a final concentration of $20 \mu \mathrm{M}$ and stored at $-80^{\circ} \mathrm{C}$ until utilization.

Approximately $20 \mathrm{pl}$ AurkA siRNA, nontargeting siRNA or Dharmacon siRNA buffer was microinjected into the cytoplasm of denuded GVI oocytes, and cells were cultured for $24 \mathrm{hr}$ in HTF/0.3\% BSA/60 $\mu \mathrm{M}$ IBMX to prevent meiotic progression and to allow for siRNA processing. The meiotic inhibitor IBMX was removed after triple washing in $\mathrm{HTFH}+$ $0.3 \%$ BSA media, and oocytes were allowed to proceed with meiosis in $\mathrm{HTF}+0.3 \%$ BSA in a $5 \% \mathrm{CO}_{2}$ incubator for $2 \mathrm{hr}$ 
to assess germinal vesicle breakdown (GVBD), $7 \mathrm{hr}$ to assess metaphase I (MI) development, and $17 \mathrm{hr}$ to categorize metaphase II (MII) development. Data were collected from three different individual experiments. The number of GVBD and MII oocytes was calculated and analyzed statistically.

\section{Total RNA Isolation, Reverse Transcription, and Real-time PCR}

Total RNA was extracted from 20 oocytes in buffer, nontargeting siRNA and AurkA siRNA microinjected groups respectively using Picopure RNA Isolation kit (Arcturus Bioscience, Mountain View, CA) following manufacturer's instructions. Oocyte cDNA was synthesized using $125 \mathrm{pmol}$ random hexamer, $500 \mathrm{mM}$ dNTP, 20 IU RNase inhibitor and 62.5 IU MultiScribe ${ }^{\mathrm{TM}}$ reverse transcriptase ( $\mathrm{ABI}$ systems) in a final volume of $50 \mu \mathrm{l}$. Primers for mouse AurkA, AurkB, and AurkC were designed with no sequence overlap between isoforms (Swain et al., 2008). Real-time PCR was performed on Applied Biosystems 7300 Real-Time PCR system using SYBR Green PCR Master Mix (Applied BioSystems, Foster City, CA). Each PCR reaction was performed with 1.5 oocyte equivalents of cDNA. Real-time PCR reactions were carried out for 40 cycles $\left(95^{\circ} \mathrm{C}\right.$ for $15 \mathrm{sec}, 60^{\circ} \mathrm{C}$ for $1 \mathrm{~min}$ ) after initial $10 \mathrm{~min}$ incubation at $95^{\circ} \mathrm{C}$. Data were collected over three replicates with triplicate samples for each isoform. Fold decreases were based on the transcript level of AurkA in siRNA microinjected oocytes, which were normalized to 1 . Comparative $\mathrm{Ct}$ method was used for data analysis and $\beta$-actin was used for internal control. Statistical significance was determined using the unpaired Student's t-test.

\section{Western Blot Analysis}

Western blot analysis was performed to determine the effects of AurkA siRNA on the protein expression levels of AURKA and other downstream signaling molecules. Because nontargeted siRNA and vehicle microinjection were similar in lack of influence on Aurk expression and meiotic progression, subsequent studies were performed with vehicle microinjection as control. Equal numbers of MI and MII oocytes in control and AurkA knockdown groups were compared. Oocytes were placed in Laemmli Sample Buffer (Bio-Rad Laboratories, Hercules, CA) according to manufacturer's instruction, vortexed and placed on ice for $15 \mathrm{~min}$. Following sonication on ice for $10 \mathrm{sec}$, samples were denatured at $90^{\circ} \mathrm{C}$ for $10 \mathrm{~min}$ and loaded on $12 \%$ gel for electrophoresis. Total protein from equal numbers of mouse oocytes in control and AurkA knockdown groups was loaded in each lane and separated by onedimensional SDS-PAGE. Gels were equilibrated and transferred to Hybond-P PVDF transfer membrane (Amersham Life Sciences, Little Chalfont Buckinghamshire, UK) by Semi-Dry Electrophoretic Transfer Cell (Bio -Rad Laboratories) according to the manufacturer's instructions. Blots were blocked in $5 \%$ nonfat milk in Tris-buffered saline (TBS) with $0.1 \%$ Tween (TBST) at room temperature for $1 \mathrm{hr}$, and incubated with the appropriate primary antibody diluted in TBST $/ 5 \%$ BSA at least overnight at $4^{\circ} \mathrm{C}$ with agitation. The antibodies included anti-AURKA (1:500, Cell Signaling, Danvers, MA), anti-phospho-ser10-histone H3 (1:500, Cell Signaling), anti-phospho-ser 28-histone H3 (1:500, Upstate, Billerica, MA), anti-ERK-2 (1:1,000, Santa Cruz, Santa Cruz, CA) and anti-GAPDH $(1: 1,000$, Novus, Littleton, CO). After complete washing in TBST, blots were incubated with appropriate peroxidase-conjugated IgG secondary antibody (Amersham Life Sciences) at room temperature for $2 \mathrm{hr}$, washed in TBST and developed with ECL Plus reagents (Amersham Life Sciences) according to the manufacturer's instructions. Membranes were re-probed using different primary antibodies after stripping at $50^{\circ} \mathrm{C}$ for $30 \mathrm{~min}$ in stripping buffer (62.5 M Tris- $\mathrm{HCl}$, pH 6.7, 2\% SDS, and $100 \mathrm{mM} \beta$-mercaptoethanol).

\section{Immunocytochemistry and Confocal Microscopic Analysis}

To examine the effect of AurkA siRNA on spindle formation, MTOC localization and chromosomal alignment, MI oocytes in control and knockdown groups were attached to poly-lysine coated coverslips separately, fixed in $2 \%(\mathrm{w} / \mathrm{v})$ paraformaldehyde for $1 \mathrm{hr}$ at $37^{\circ} \mathrm{C}$, blocked overnight with PBST $/ 0.3 \%$ BSA at $4{ }^{\circ} \mathrm{C}$ and incubated with primary antibodies at $37^{\circ} \mathrm{C}$ for $1 \mathrm{hr}$. Antibodies used for experiments included anti- $\beta$-tubulin to visualize microtubules (Sigma, 1:200); anti-pericentrin (Covance, Princeton, NJ, 1:200) and $\gamma$-tubulin (Sigma, 1:100) to visualize MTOCs. Chromatin was stained with Hoechst $33342(5 \mu \mathrm{g} / \mathrm{ml})$. After washing of primary antibodies, samples were reacted with the appropriate Alexa568 and 488 conjugated secondary antibodies (Molecular Probes, Carlsbad, CA) at a 1:1,000 dilution for $1 \mathrm{hr}$ at $37^{\circ} \mathrm{C}$. Coverslips were then mounted on glass slides for visualization under $1,000 \times$ confocal microscope (Olympus Fluoview 500). For confocal microscopic analysis, Z-stacks were captured with $0.3 \mu \mathrm{m}$ steps through the entire spindle, MTOC, and chromosome area in each oocyte, then images were reconstituted via Fluoview software. Images were taken and graded in blinded fashion as normal or abnormal. Criteria included normal shaped barrel spindle formation, proper localization of chromatin on the metaphase plate, and proper localization of MTOC proteins (pericentrin and $\gamma$-tubulin) at the spindle poles.

\section{Chromosome Analysis}

Metaphase II oocytes matured for $17 \mathrm{hr}$ in control and knockdown groups were collected and chromosomal spreads prepared using $1 \%$ paraformaldehyde method (Hodges and Hunt, 2002) with slight modification. All treatments from the same day were prepared side-by-side to control for variability. Briefly, zona pellucidae of oocytes were removed by $0.6 \%$ proteinase $\mathrm{K}$ in PBS. Zona-free oocytes were then washed, treated with hypotonic buffer $(0.075 \mathrm{M} \mathrm{KCl})$ for $5 \mathrm{~min}$, and fixed onto a microscope slide dipped in the solution of $1 \%$ paraformaldehyde/3 mM DTT/ $0.15 \%$ TritonX-100 ( $\mathrm{pH}$ 9.2). Slides were then placed into 
humidified chambers overnight, air dried at room temperature, stained by $5 \mu \mathrm{g} / \mathrm{ml}$ Hoechst 33342 for $5 \mathrm{~min}$, triple washed with $1 \times$ PBS and mounted in glycerol mounting solution. Chromosomal spreads were analyzed at $1,000 \times$ on a Leica DMR microscope and images taken so that the chromosome numbers could be counted in a treatment blinded fashion. Numbers of chromosomes were counted to determine segregation errors and data was statistically analyzed.

\section{Co-Immunoprecipitation}

To determine if AURKA was directly involved in the phosphorylation of histone $\mathrm{H} 3$ under physiological condition in cells and oocytes, co-immunoprecipitation experiments using phospho-histone H3 (ser10) antibody (Cell Signaling) were performed in MII oocytes and the human granulosalike tumor cell line, KGN, following manufacture's instruction. Briefly, MII oocytes $(n=566)$ were collected from superovulated CF1 mice ( 8 weeks old) and KGN cells cultured in $75 \mathrm{~cm}^{2}$ flask were harvested at their $90 \%$ confluence. Both cells were triple washed with cold $1 \times$ PBS before transferring to or scrapping off in ice cold $1 \times$ cell lysis buffer [20 mM Tris ( $\mathrm{pH} 7.5$ ), $150 \mathrm{mM} \mathrm{NaCl}, 1 \mathrm{mM}$ EDTA, $1 \mathrm{mM}$ EGTA, 1\% Triton X-100, $2.5 \mathrm{mM}$ Sodium pyrophosphate, $1 \mathrm{mM} \beta$-glycerophosphate, $1 \mathrm{mM} \mathrm{Na}_{3} \mathrm{VO}_{4}$ with proteinase inhibitor cocktail (Roche, Indianapolis, IN)]. Cells were sonicated on ice five times for $10 \mathrm{sec}$ each, centrifuged at $12,000 \mathrm{~g}$ for $10 \mathrm{~min}$ at $4^{\circ} \mathrm{C}$, and their supernatants were recovered for co-IP experiment. The supernatants were divided into two equal parts and $1 \times$ PBS or phosphoHistone $\mathrm{H} 3$ (ser10) antibody were added, respectively, in control or Co-IP groups (1:50 dilution), and incubated overnight at $4{ }^{\circ} \mathrm{C}$ with gentle rocking. The following day, prewashed protein A agarose beads (50\% slurry, Millipore, Billerica, MA) were added into each group, incubated at $4^{\circ} \mathrm{C}$ with gentle rocking for $3 \mathrm{hr}$, then pelleted down at 12,000 for $30 \mathrm{sec}$ at $4^{\circ} \mathrm{C}$. The eluted supernatants were kept for Western blot analysis later and protein $\mathrm{A}$ agarose beads pellets were washed with $500 \mu$ l lysis buffer for four times at $4^{\circ} \mathrm{C}$, then $3 \times$ SDS sample buffer added and heated at $95-100^{\circ} \mathrm{C}$ for $5 \mathrm{~min}$. Western blot analysis was carried out as described. The whole KGN cell lysate was served as non -IP control. Experiments were repeated twice.

\section{Statistical Analysis}

Unpaired Student $t$-test and Chi-square analysis was performed, as indicated in results, table or the figure legends, using GraphPad Software. Differences of $P<0.05$ were considered to be significant.

\section{ACKNOWLEDGMENTS}

Authors would like to thank the Transgenic Animal Model Core Facility at the University of Michigan for providing the microinjection system and technical support.

\section{REFERENCES}

Anand S, Penrhyn-Lowe S, Venkitaraman A. 2003. AURORO-A amplification overrides the mitotic spindle assembly checkpoint, inducing resistance to Taxol. Cancer Cells 3:51-62.

Barr A, Gergely F. 2007. Aurora-A: The maker and breaker of spindle poles. J Cell Sci 120:2987-2996.

Bui H, Yamaoka E, Miyano T. 2004. Involvement of histone H3 (Ser10) phosphorylation in chromosome condensation without Cdc2 kinase and mitogen-activated protein kinase activation in pig oocytes. Biol Reprod 70:1843-1851.

Chiang T, Duncan F, Schindler K, Schultz R, Lampson M. 2010. Evidence that weakened centromere cohesion is a leading cause of age-related aneuploidy in oocytes. Curr Biol 20: 1522-1528.

Cleveland D, Mao Y, Sullivan K. 2003. Centromeres and kinetochores: From epigenetics to mitotic checkpoint signaling. Cell 112:407-421.

Crane R, Gadea B, Littlepage L, Wu H, Ruderman J. 2003. Aurora A, meiosis and mitosis. Biol Cell 96:215-229.

Crosio C, Fimia G, Loury R, Kimura M, Okano Y, Zhou H, Sen S, Allis C, Sassone-Corsi P. 2002. Mitotic phosphorylation of histone H3: Spatio-temporal regulation by mammalian Aurora kinases. Mol Cell Biol 22:874-885.

Ditchfield C, Johnson V, Tighe A, Ellston R, Haworth C, Johnson T, Mortlock A, Keen N, Taylor S. 2003. Aurora B couples chromosome alignment with anaphse by targeting BubR1, Mad2, and Cenp-E to kinetochores. J Cell Biol 161:267-280.

Ducat D, Zhen Y. 2004. Aurora kinases in spindle assembly and chromosome segregation. Exp Cell Res 301:60-67.

Duncan F, Chiang T, Schultz R, Lampson M. 2009. Evidence that a defective spindle assembly checkpoint is not the primary cause of maternal age-associated aneuploidy in mouse eggs. Biol Reprod 81:768-776.

Eichenlaub-Ritter U. 2009. Aurora kinase B, epigenetic state of centromeric heterochromatin and chiasma resolution in oocytes. Reprod BioMed Online 19:352-368.

Fire A, Xu S, Montgomery M, Kostas S, Driver S, Mello C. 1998. Potent and specific genetic interference by double-stranded RNA in Caenorhabditis elegans. Nature 391:806-811.

Goto H, Yasui Y, Kawajiri A, Nigg E, Terada Y, Tatsuka M, Nagata $\mathrm{K}$, Inagaki M. 2003. Aurora-B regulates the cleavage furrospecific vimentin phosphorylation in the cytokinetic process. $J$ Biol Chem 278:8526-8530.

Hammond S, Bernstein E, Beach D, Hannon G. 2000. An RNAdirected nuclease mediates post-transcriptional gene silencing in Drosophila cells. Nat Biotechnol 404:293-296.

Hodges C, Hunt P. 2002. Simultaneous analysis of chromosomes and chromosome-associated proteins in mamalian oocytes and embryos. Chromosoma 111:165-169. 
Hsu J, Sun Z, Li X, Reuben M, Tatchell K, Bishop D, Grushcow J, Brame C, Caldwell J, Hunt D, Lin R, Smith M, Allis C. 2000. Mitotic phosphorylation of histone $\mathrm{H} 3$ is governed by Ipl1/aurora kinase and GLC7/PP1 phosphatase in budding yeast and nematodes. Cell 102:279-291.

Hunt $\mathrm{P}$, Koehler K, Susiarjo M, Hodges C, llagan A, Voigt R, Thomas S, Thomas B, Hassold T. 2003. Bisphenol A exposure causes meiotic aneuploidy in the female mouse. Curr Biol 13:546-553.

Jelinkova L, Kubelka M. 2006. Neither Aurora B activity nor histone $\mathrm{H} 3$ phosphorylation is essential for chromosome condensation during meiotic maturation of porcine oocytes. Biol Reprod 74: 905-912.

Kunitoku N, Sasayama T, Marumoto T, Zhang D, Honda S, Kobayashi O, Hatakeyama K, Ushio Y, Saya H, Hirota T. 2003. CENP-A phosphorylation by Aurora-A in prophase is required for enrichment of Aurora-B at inner centromeres and for kinetochore function. Dev Cell 5:853-864.

Lane S, Chang H, Jennings P, Jones K. 2010. The Aurora kinase inhibitor ZM447439 accelerates first meiosis in mouse oocytes by overriding the spindle assembly checkpoint. Reproduction 140:521-530

LiX, Sakashita G, Matsuzaki H, Sugimoto K, Kimura K, Hanaoka F, Taniguchi H, Furukawa K, Urano T. 2004. Direct association with inner centromere protein (INCENP) activates the novel chromosomal passenger protein, Aurora-C. J Biol Chem 279: 47201-47211.

Liu L, Aoki V, Carrell D. 2008. Evaluation of the developmental competence and chromosomal compliment of mouse oocytes derived from in-vitro growth and maturation of preantral follicles. J Assist Reprod Genet 25:107-113.

Lu L, Wood J, Ye L, Minter-Dykhouse K, Saunders T, YuX, Chen J. 2008. Aurora $A$ is essential for early embryonic development and tumor suppression. J Biol Chem 278:8526-8530.

Marumoto T, Zhang D, Saya H. 2005. Aurora-A-A guardian of poles. Nat Rev Cancer 5:42-50.

Meister G, Tuschl T. 2004. Mechanisms of gene silencing by double-stranded RNA. Nature 431:343-349.

Mello C, Conte D, Jr. 2004. Revealing the world of RNA interface. Nature 431:338-342.

Meraldi P, Honda R, Nigg E. 2004. Aurora kinases link chromosome segregation and cell division to cancer susceptibility. Curr Opin Genet Dev 14:29-36.

Reis A, Madgwick S, Change H, Nabti I, Levasseur M, Jones K. 2007. Prometaphase APC ${ }^{\text {cdh1 }}$ activity prevents non-disjunction in mammalian oocytes. Nat Cell Biol 9:1192-1198.

Roberts R, latropoulou A, Ciantar D, Stark J, Becker D, Franks S, Hardy K. 2005. Follicle-stimulating hormone affects metaphase I chromosome alignment and increases aneuploidy in mouse oocytes matured in vitro. Biol Reprod 72:107118.
Saskova A, Solc P, Baran V, Kubelka M, Schultz M, Motlik J. 2008. Aurora kinase A controls meiosis I progression in mouse oocytes. Cell Cycle 7:2368-2376.

Sato F, Marrs R. 1986. The effect of pregnant mare serum gonadotropin on mouse embryos fertilized in vivo or in vitro. $\mathrm{J}$ In Vitro Fert Embryo Transf 3:353-357.

Schuh M, Ellenberg J. 2007. Self-organization of MTOCs replaces centrosome function during acentrosomal spindle assembly in live mouse oocytes. Cell 130:484-498.

Shuda K, Schindler K, Ma J, Schultz R, Donovan P. 2009. Aurora kinase $B$ modulates chromosome alignment in mouse oocytes. Mol Reprod Dev 76:1094-1105.

Solc P, Saskova A, Baran V, Kubelka M, Schultz R, Motlik J. 2008. CDC25A phosphatase controls meiosis I progression in mouse oocytes. Dev Biol 317:260-269.

Svoboda P, Stein P, Hayashi H, Schultz R. 2000. Selective reduction of dormant maternal mRNA in mouse oocytes by RNA interference. Development 127:4147-4156.

Swain J, Ding J, Brautigan D, Villa-Moruzzi E, Smith G. 2007. Proper chromatin condensation and maintenance of histone $\mathrm{H} 3$ phosphorylation during mouse oocyte meiosis requires protein phosphatase activity. Biol Reprod 76:628-638.

Swain J, Ding J, Wu J, Smith G. 2008. Regulation of spindle and chromatin dynamics during early and late stages of oocyte maturation of aurora kinases. Mol Hum Reprod 14:291-299.

Ulisse S, Delcros J, Baldini E, Toller M, Curcio F, Giacomelli L, Prigent C, Ambesi-Impiobato F, D'Armiento M, Arlot-Bonnemains Y. 2006. Expression of Aurora kinases in human thyroid carcinoma cell lines and tissues. Int J Cancer 119:275-282.

Uzbekova S, Arlot-Bonnemains Y, Dupont J, Dalbiès-Tran R, Papillier P, Pennetier S, Thélie A, Perreau C, Mermillod P, Prigent C, Uzbekov R. 2008. Spatio-temporal expression patterns of aurora kinases A, B, and C and cytoplasmic polyadenylation-element-binding protein in bovine oocytes during meiotic maturation. Biol Reprod 78:218-233.

Wang Q, Wang C, Ai J, Xiong B, Yin S, Hou Y, Chen D, Schatten H, Sun Q. 2006. Histone phosphorylation and pericentromeric histone modifications in oocyte meiosis. Cell Cycle 5: 1974-1982.

Wang J, Lei Z, Nan C, Yin S, Hou Y, Li Y, Chen D, Sun Q. 2007. RNA interference as a tool to study the function of MAD2 in mouse oocyte meiotic maturation. Mol Reprod Dev 74:116-124.

Wei Y, Yu L, Bowen J, Gorovsky M, Allis C. 1999. Phosphorylation of histone $\mathrm{H} 3$ is required for proper chromosome condensation and segregation. Cell 97:99-109.

Wianny F, Zernicka-Goetz M. 2000. Specific interference with gene function by double-stranded RNA in early mouse development. Nat Cell Biol 2:70-75.

Yan X, Cao L, Li Q, Wu Y, Zhang H, Saiyin H, Liu X, Zhang X, Shi Q, Yu L. 2005. Aurora $C$ is directly associated with survivin and required for cytokinesis. Genes Cells 10:617-626. 
Yang K, Li S, Chang C, Tang C, Lin Y, Lee S, Tang T. 2010. Aurora$\mathrm{C}$ kinase deficiency causes cytokinesis failure in meiosis I and production of large polyploid oocytes in mice. Cell Cycle 21:2371-2383.

Yao L, Zhong Z, Zhang L, Chen D, Schatten H, Sun Q. 2004. Aurora-A is a critical regulator of microtubule assembly and nuclear activity in mouse oocytes, fertilized eggs, and early embryos. Biol Reprod 70:1392-1399.

Zeitlin S, Barber C, Allis C, Sullivan K. 2000. Differential regulation of CENP-A and histone $\mathrm{H} 3$ phosphorylation in G2/M. J Cell Sci 114:653-661. 